\title{
Soliton propagation in a medium with Kerr nonlinearity and resonant impurities: A variational approach
}

\author{
D. P. Caetano, S. B. Cavalcanti, and J. M. Hickmann \\ Departamento de Física, Universidade Federal de Alagoas, Cidade Universitária, AL 57072-970 Maceió, Brazil \\ A. M. Kamchatnov \\ Institute of Spectroscopy, Russian Academy of Sciences, Troitsk 142190, Moscow Region, Russia \\ R. A. Kraenkel and E. A. Makarova \\ Instituto de Física Teórica, Universidade Estadual Paulista-UNESP, Rua Pamplona 145, 01405-900 São Paulo, Brazil
}

(Received 9 December 2002; published 25 April 2003)

\begin{abstract}
Using a variational approach we have studied the shape preserving coherent propagation of light pulses in a resonant dispersive medium in the presence of the Kerr nonlinearity. Within the framework of a combined nonintegrable system composed of one nonlinear Schrödinger and a pair of Bloch equations, we show the existence of a solitary wave. We have tested our analytical solution through numerical simulations confirming its solitary wave nature.
\end{abstract}

DOI: 10.1103/PhysRevE.67.046615

PACS number(s): 42.65.Tg, 42.50.-p, 42.81.Dp

\section{INTRODUCTION}

Investigation on coherent pulse propagation in a fiber waveguide with resonant impurities is a recurrent subject in the physical literature [1-3]. It combines two quite different regimes of soliton propagation: resonant propagation related to the self-induced transparency (SIT) phenomenon and nonresonant propagation under the influence of weak dispersion and Kerr-like nonlinearity described by a mean field nonlinear Schrödinger equation (NLS). Recently, [4] periodic and soliton solutions describing the propagation of an optical field through a nonlinear dispersive medium embedded with two level atoms, were obtained within the inverse scattering transform (IST) method. However, the family of mixed soliton solutions presented in these studies is only possible for a particular choice of pulse power satisfying both the NLS condition for the fundamental soliton as well as the area theorem for the SIT soliton. More specifically, the power required to launch a fundamental fiber soliton should be equal to the power of a $2 \pi$-SIT pulse, i.e., $P_{2 \pi}=P_{N=1}$. This condition on the interdependence of SIT and Kerr parameters is a physical consequence of the condition of complete integrability of the equations. Unfortunately this fact limits severely the usefulness of the SIT-Kerr soliton for practical applications [5]. Hence it is important to develop some approximate alternative approach to the IST to deal with a nonintegrable system so that this limitation is weakened or eliminated at all. This is a perfect scenario for a variational method used successfully in perturbation theory of solitons [6], propagation in the presence of nonlinear dissipation [7], nonintegrable systems such as cascaded $\chi^{(2)}$ nonlinearity $[8,9]$, etc. In these references, an approximate variational family of solitons was obtained, convergent to the known exact solutions for particular values of the physical parameters. Usually, the variational result reproduces interpolation formulas for solitonlike pulses in a wide region of parameters between limiting regions of values that admit exact solutions.
Based on the above discussion, we apply the variational method to investigate the existence of solitary wave solutions in media with combined Kerr nonlinearity and SIT resonance under conditions that do not necessarily satisfy the integrability requirement. Using an ansatz based on the exact result we have found a solitary wave solution whose new features are described by an extra term. This solution is shown to converge to the exact result obtained previously by the inverse scattering method. We show further that the analytical result is confirmed by numerical simulations of the NLS-Bloch equations describing the system evolution.

\section{RESONANT AND NONRESONANT LAGRANGIAN}

We begin by writing the equations for a system with combined Kerr and SIT terms in a standard form

$$
\begin{gathered}
i E_{Z}+E_{T T}+2 g|E|^{2} E+d=0, \\
d_{T}+2 i \Delta d=-2 i f E n, \\
n_{T}=i f\left(d^{*} E-d E^{*}\right) .
\end{gathered}
$$

Here $T=t-z . Z$ and $t$ are the dimensionless spatial and time coordinates, respectively; $E(Z, T)$ is the slowly varying electromagnetic field envelope. $d(Z, T)$ and $n(Z, T)$ denote the normalized electric dipole moment of the transition and the population difference of the two-level atoms, respectively. $\Delta$ is the detuning parameter between the atomic transition and the central frequency of the electromagnetic wave. The variables $d$ and $n$ satisfy the normalization condition

$$
|d|^{2}+n^{2}=1,
$$

which reflects the conservation of probability in the sense that the total probability for an atom to be found either in the upper or lower levels is equal to unity.

Because of the condition set by Eq. (2), the Lagrangian for the system cannot be written directly in terms of the 
variables $E, d, n$. To circumvent this difficulty we introduce the real variables $E_{1}, E_{2}, \theta, \phi$ according to

$$
E=E_{1}-i E_{2}, \quad d=\sin \theta e^{-i \phi}, \quad n=\cos \theta,
$$

so that Eq. (2) is satisfied automatically and the system of Eqs. (1) takes the form

$$
\begin{gathered}
E_{1, Z}-E_{2, T T}-2 g\left(E_{1}^{2}+E_{2}^{2}\right) E_{2}-\sin \theta \sin \phi=0, \\
E_{2, Z}+E_{1, T T}+2 g\left(E_{1}^{2}+E_{2}^{2}\right) E_{1}+\sin \theta \cos \phi=0, \\
\sin \theta\left(\phi_{T}-2 \Delta\right)-2 f \cos \theta\left(E_{1} \sin \phi+E_{2} \sin \phi\right)=0, \\
\theta_{T}+2 f\left(-E_{1} \sin \phi+E_{2} \cos \phi\right)=0 .
\end{gathered}
$$

The Lagrangian form of these last two equations was indicated in Ref. [10]. Using this result we obtain the full Lagrangian of the system in the form

$$
\begin{aligned}
\mathcal{L}= & \frac{1}{2}\left(E_{1, Z} E_{2}-E_{1} E_{2, Z}\right)+\frac{1}{2}\left(E_{1, T}^{2}+E_{2, T}^{2}\right)-\frac{g}{2}\left(E_{1}^{2}+E_{2}^{2}\right) \\
& -\frac{1}{2 f}\left(\phi_{T}-2 \Delta\right) \cos \theta-\sin \theta\left(E_{1} \cos \phi+E_{2} \sin \phi\right) .
\end{aligned}
$$

It is easy to check that the Lagrange equations corresponding to the field Lagrangian Eq. (4) can be reduced to the system of equations (3). In original complex variables this Lagrangian becomes

$$
\begin{aligned}
\mathcal{L}= & -\frac{i}{2}\left(E_{Z} E^{*}-E E_{Z}^{*}\right)+\left|E_{T}\right|^{2}-g|E|^{4}-E d^{*}-E^{*} d \\
& -\frac{1}{f}\left[\frac{i\left(d_{T} d^{*}-d d_{T}^{*}\right)}{2|d|^{2}}-2 \Delta\right] n .
\end{aligned}
$$

It is known that the combined system composed of the NLS equation together with the SIT equations under the condition

$$
g=f^{2}
$$

has an exact simultaneous soliton solution which can be written as [4]

$$
\begin{aligned}
E(T, Z)= & \frac{2 \gamma}{f} \exp \left[-2 i \alpha T-i\left(4\left(\alpha^{2}-\gamma^{2}\right)\right.\right. \\
& \left.\left.+\frac{f(\alpha-\Delta)}{(\alpha-\Delta)^{2}+\gamma^{2}}\right) Z\right] \frac{1}{\cosh [2 \gamma(T+Z / V)]}
\end{aligned}
$$

$$
\begin{aligned}
d(T, Z)= & -\frac{2 \gamma}{(\alpha-\Delta)^{2}+\gamma^{2}} \frac{\alpha-\Delta+i \gamma \tanh [2 \gamma(T+Z / V)]}{\cosh [2 \gamma(T+Z / V)]} \\
& \times \exp \left[-2 i \alpha T-i\left(4\left(\alpha^{2}-\gamma^{2}\right)\right.\right. \\
& \left.\left.+\frac{f(\alpha-\Delta)}{(\alpha-\Delta)^{2}+\gamma^{2}}\right) Z\right], \\
n(T, Z)= & \frac{2 \gamma^{2}}{(\alpha-\Delta)^{2}+\gamma^{2}} \frac{1}{\cosh ^{2}[2 \gamma(T+Z / V)]}-1,
\end{aligned}
$$

where the soliton's velocity $V$ is given by

$$
\frac{1}{V}=4 \alpha-\frac{f}{2\left[(\alpha-\Delta)^{2}+\gamma^{2}\right]},
$$

with $\alpha$ and $\gamma$ being free parameters that determine the shape of the soliton and its velocity. Our aim is to generalize system (7) for arbitrary values of $g$ and $f$.

\section{VARIATIONAL APPROACH TO SOLITON PROPAGATION}

Following the variational approach, (see Refs. [6-9]) we choose a realistic and yet simple set of trial functions of $T$ and then calculate the "averaged" Lagrangian

$$
L=\int_{-\infty}^{\infty} \mathcal{L} d T
$$

to determine the dependence of the parameters entering into the trial function on the evolution variable $Z$. It is well known that the success of this approach depends crucially on a proper choice of trial functions. For example, frequently used Gaussian pulses simplify the calculation of integrals in Eq. (9), but they cannot reproduce exact solutions such as those in Eqs. (7). A natural choice here for the trial functions, is to use the exact solution with varying parameters such as the following:

$$
\begin{gathered}
n(T, Z)=\frac{2 C^{2}}{B^{2}+C^{2}} \frac{1}{\cosh ^{2}[\kappa(T+\zeta)]}-1, \\
d(T, Z)=-\frac{2 C}{B^{2}+C^{2}}\{B+i C \tanh [\kappa(T+\zeta)]\} \\
\times \frac{\exp (i \phi)}{\cosh [\kappa(T+\zeta)]}, \\
E(T, Z)=\frac{A \exp (i \phi)}{\cosh [\kappa(T+\zeta)]},
\end{gathered}
$$

where

$$
\phi=\omega(T+\zeta)+\eta
$$


and $A(Z), B(Z), C(Z), \zeta(Z), \eta(Z), \kappa(Z), \omega(Z)$ are all considered as functions of $Z$. The form of $d$ and $n$ is chosen so that Eq. (2) is satisfied automatically.

Substituting these trial functions in the Lagrangian, one easily finds the exact soliton solution of the bare NLS equation (without SIT terms). However, a more extensive calculation shows that the SIT soliton solution is not reproduced exactly by this choice of trial functions. Fortunately, the same calculation indicates that this deficiency may be fixed easily by a simple change of a constant factor in one of the terms in the averaged Lagrangian. As a result we obtain our Lagrangian final form

$$
\begin{aligned}
\mathcal{L}= & \frac{2 A^{2}}{\kappa}\left(\omega \zeta_{Z}+\eta_{Z}\right)+\frac{2}{3} \kappa A^{2}+2 \frac{A^{2} \omega^{2}}{\kappa}-\frac{4}{3} \frac{g A^{4}}{\kappa} \\
& +\frac{8 A B C}{\kappa\left(B^{2}+C^{2}\right)}+\frac{4}{f} \frac{\omega+2 \Delta}{\kappa} \frac{C^{2}}{B^{2}+C^{2}} \\
& -\frac{4}{f} \frac{B C}{B^{2}+C^{2}}+\frac{4}{f} \arctan \frac{C}{B} .
\end{aligned}
$$

To show that this Lagrangian is a correct starting point for the derivation of interpolation formulas for soliton solutions, we have to prove that it leads to exact soliton solutions in the special cases when such solutions exist. For the sake of clarity, let us consider them separately.

\section{NLS LIMIT}

In this limit the Lagrangian described in Eq. (11) reduces to

$$
\mathcal{L}=\frac{2 A^{2}}{\kappa}\left(\omega \zeta_{Z}+\eta_{Z}\right)+\frac{2}{3} \kappa A^{2}+2 \frac{A^{2} \omega^{2}}{\kappa}-\frac{4}{3} \frac{g A^{4}}{\kappa} .
$$

Lagrange equations

$$
\frac{\partial L}{\partial A}=\frac{\partial L}{\partial \kappa}=\frac{\partial L}{\partial \omega}=0, \quad \frac{\partial}{\partial Z} \frac{\partial L}{\partial \zeta_{Z}}=\frac{\partial}{\partial Z} \frac{\partial L}{\partial \eta_{Z}}=0
$$

yield to

$$
\begin{gathered}
\omega \zeta_{Z}+\eta_{Z}+\omega^{2}+\frac{1}{3} \kappa^{2}-\frac{4}{3} g A^{2}=0, \\
\omega \zeta_{Z}+\eta_{Z}+\omega^{2}-\frac{1}{3} \kappa^{2}-\frac{2}{3} g A^{2}=0, \\
\zeta_{Z}=-2 \omega, \\
\frac{\partial}{\partial Z}\left(\frac{A^{2} \omega}{\kappa}\right)=\frac{\partial}{\partial Z}\left(\frac{A^{2}}{\kappa}\right)=0 .
\end{gathered}
$$

The difference between Eqs. (14) and (15) gives directly

$$
A=\frac{\kappa}{\sqrt{g}}
$$

and this relation together with Eq. (17) show that $A, \kappa$, and $\omega$ are constants as expected. Then Eqs. (14) and (16) give $\eta_{Z}=\kappa^{2}+\omega^{2}$, that is

$$
\zeta=-2 \omega Z, \eta=\left(\kappa^{2}+\omega^{2}\right), \omega \zeta+\eta=\left(\kappa^{2}-\omega^{2}\right) Z .
$$

Thus, we can write the trial function Eq. (10) as

$$
E(T, Z)=\frac{\kappa}{\sqrt{g}} \frac{\exp \left[i\left(\omega T+\left(\kappa^{2}-\omega^{2}\right) Z\right]\right.}{\cosh [\kappa(T-2 \omega Z)]},
$$

or after introducing new parameters

$$
\kappa=2 \gamma, \omega=-2 \alpha,
$$

we arrive at the following expression:

$$
E(T, Z)=\frac{2 \gamma}{\sqrt{g}} \frac{\exp \left[-2 i \alpha T-4 i\left(\alpha^{2}-\gamma^{2}\right) Z\right]}{\cosh [2 \gamma(T+4 \alpha Z)]}
$$

which coincides exactly with the corresponding limit of Eq. (7).

\section{SIT LIMIT}

In this limit Lagrangian (11) takes the form

$$
\begin{aligned}
L= & \frac{8 A B C}{\kappa\left(B^{2}+C^{2}\right)}+\frac{4}{f} \frac{\omega+2 \Delta}{\kappa} \frac{C^{2}}{B^{2}+C^{2}} \\
& -\frac{4}{f} \frac{B C}{B^{2}+C^{2}}+\frac{4}{f} \arctan \frac{C}{B} .
\end{aligned}
$$

Now, Lagrange equations

$$
\frac{\partial L}{\partial A}=\frac{\partial L}{\partial B}=\frac{\partial L}{\partial C}=\frac{\partial L}{\partial \kappa}=\frac{\partial L}{\partial \omega}=0, \quad \frac{\partial}{\partial Z} \frac{\partial L}{\partial \zeta_{Z}}=\frac{\partial}{\partial Z} \frac{\partial L}{\partial \eta_{Z}}=0
$$

yield

$$
\begin{gathered}
\omega \zeta_{Z}+\eta_{Z}=-\frac{2 B C}{A\left(B^{2}+C^{2}\right)} \\
f A\left(B^{2}-C^{2}\right)+(\omega+2 \Delta) B C+\kappa C^{2}=0 \\
\zeta_{Z}=-\frac{2}{f} \frac{C^{2}}{A^{2}\left(B^{2}+C^{2}\right)} \\
f\left(\omega \zeta_{Z}+\eta_{Z}\right) A^{2}\left(B^{2}+C^{2}\right)+4 f A B C+2(\omega+2 \Delta) C^{2}=0 \\
\frac{A^{2}}{\kappa}=\text { const, } \frac{\omega A^{2}}{\kappa}=\text { const }
\end{gathered}
$$

where Eq. (25) is obtained twice. Substitution of Eq. (24) into Eq. (27) gives 


$$
\frac{A B}{C}=-\frac{\omega+2 \Delta}{f},
$$

and hence from Eq. (25) we get

$$
A=\frac{\kappa}{f} .
$$

This equation together with Eq. (28) show again that $A, \kappa, \omega$ are constant. Introducing $\alpha$ and $\gamma$ via Eq. (20), we find that the other equations are satisfied if

$$
A=\frac{2 \gamma}{f}, \quad B=2(\alpha-\Delta), \quad C=2 \gamma
$$

and then the soliton velocity is equal to

$$
\zeta_{Z}=-\frac{f}{2\left[(\alpha-\Delta)^{2}+\gamma^{2}\right]} .
$$

Substitution of these parameters into the trial function [Eq. (10)] produces the exact solution of the SIT equations which coincides with the corresponding limit of the combined solution [Eqs. (7)].

These calculations justify the above statement that the Lagrangian together with the trial functions provide the basis to obtain approximate formulas for the soliton solution. After gaining this experience we can proceed to the general case with arbitrary values of $g$ and $f$.

\section{COMBINED NLS AND SIT REGIME}

In the general case of Lagrangian equation (11), Hamilton's principle provides the following set of Lagrange equations:

$$
\begin{gathered}
\omega \zeta_{Z}+\eta_{Z}+\omega^{2}+\frac{1}{3} \kappa^{2}-\frac{4}{3} g A^{2}+\frac{2 B C}{A\left(B^{2}+C^{2}\right)}=0 \\
f A\left(B^{2}-C^{2}\right)+(\omega+2 \Delta) B C+\kappa C^{2}=0, \\
\zeta_{Z}=-2 \omega-\frac{2}{f} \frac{C^{2}}{A^{2}\left(B^{2}+C^{2}\right)}, \\
\omega \zeta_{Z}+\eta_{Z}+\omega^{2}-\frac{1}{3} \kappa^{2}-\frac{2}{3} g A^{2}+\frac{4 B C}{A\left(B^{2}+C^{2}\right)} \\
+\frac{2(\omega+2 \Delta)}{f} \frac{C^{2}}{A^{2}\left(B^{2}+C^{2}\right)}=0
\end{gathered}
$$

as well as Eq. (29). Again we introduce new constants $\alpha$ and $\gamma$, so that $A, B$, and $C$ are determined by Eqs. (44) and

$$
\omega=-2 \alpha+\gamma \mu
$$

where $\mu$ is the parameter to be determined. To this end, we find on one hand that the difference between Eqs. (33) and (36) gives

$$
\kappa^{2}=\frac{4 g}{f^{2}} \gamma^{2}+\frac{3 f \gamma \mu}{4\left[(\alpha-\Delta)^{2}+\gamma^{2}\right]},
$$

and on the other hand from Eq. (34) we obtain

$$
\kappa=2 \gamma-(\alpha-\Delta) \mu \text {. }
$$

Thus, we arrive at a quadratic equation for $\mu$. Note that $\mu$ $=0$ implies $g=f^{2}$ and this particular case reproduces the exact solution. Thus we obtain

$$
\mu=\frac{1}{2(\alpha-\Delta)^{2}}\left\{4(\alpha-\Delta) \gamma+\frac{3 f \gamma}{4\left[(\alpha-\Delta)^{2}+\gamma^{2}\right]}-\sqrt{\left(4(\alpha-\Delta) \gamma+\frac{3 f \gamma}{4\left[(\alpha-\Delta)^{2}+\gamma^{2}\right]}\right)^{2}-16(\alpha-\Delta)^{2}\left(1-\frac{g}{f^{2}}\right)}\right\}
$$

At last, we find the expression for soliton's velocity

$$
\frac{1}{V}=\zeta_{Z}=4 \alpha-\frac{f}{2\left[(\alpha-\Delta)^{2}+\gamma^{2}\right]}-2 \gamma \mu,
$$

where Eqs. (37) and (39) with $\mu$ defined by Eq. (40) give the width of the soliton and its velocity as functions of the ratio $g / f^{2}$ provided the amplitude of the soliton is given by

$$
A=\frac{2 \gamma}{f}
$$

\section{NUMERICAL RESULTS}

To test our analytical solutions we have carried out numerical experiments. The basic idea is to show that using the variational solution as an initial condition the resulting propagation exhibits a solitary wave behavior. To obtain the propagation behavior, we have numerically solved the set of Eqs. (1), using a combination of the Runge-Kutta with the beam propagation method to determine both the dynamics of the atomic system and the optical field evolution, with the following set of parameters: $\Delta=0.1, g=0.125$. We choose the initial conditions for the atomic system so that the population is in the ground state to start with and we choose a sech temporal profile for the optical pulse with area $2 \pi$, corresponding to a NLS soliton with amplitude $A=0.5$. In Fig. 1(a) we show the pulse propagation illustrating solitary wave behavior for the integrable case, i.e., for $g=f^{2}$. While Fig. 2 illustrates the situation for the nonintegrable case, i.e., $g=2 f^{2}$, where we can clearly see a solitary wave propagat- 


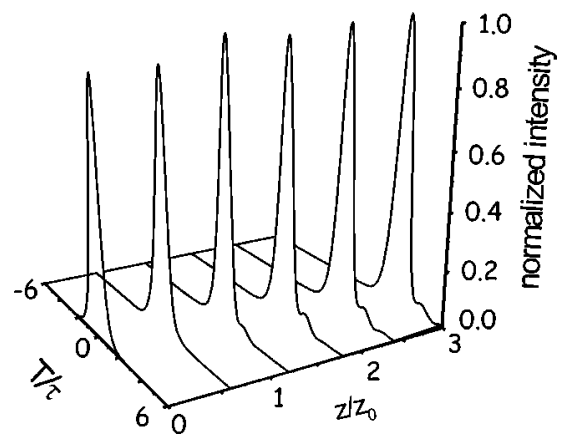

FIG. 1. Soliton propagation for the integrable case, $g=f^{2}$.

ing stably for several soliton periods.

\section{CONCLUSIONS}

Coherent soliton propagation in a resonant fiber waveguide is demonstrated for a general system of equations that do not satisfy integrability conditions, opening the real possibility of obtaining the SIT-Kerr soliton in practical conditions. Within a variational approach, we have obtained the full description of a family of approximate solutions that exhibits solitary wave properties beyond the mixed soliton state condition, that is, $P_{2 \pi} \neq P_{N=1}$. Based on the exact result for the integrable system, we were able to relax the dependence among the physical parameters allowing for realistic conditions to permit the experimental observation of a SIT-Kerr soliton. The variational solutions obtained depend on two free parameters and in the limiting cases are reduced

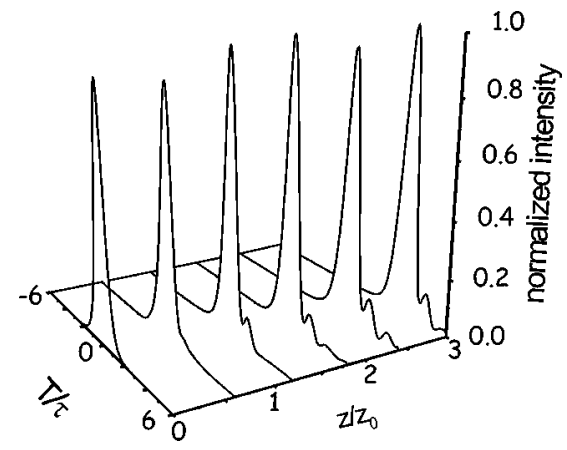

FIG. 2. Soliton propagation for the nonintegrable case, $g$ $=2 f^{2}$.

to the correspondent exact solution . Furthermore, we have confirmed the analytical results by numerical simulations, evidencing the solitary behavior of the light pulse. This SITKerr solitonic behavior in doped fiber media may be proved extremely useful to the future of optical communications and photonic devices. It combines the convenient propagation properties of an optical fiber with the remarkable manipulation properties of a resonant active medium opening a new perspective for optical techniques.

\section{ACKNOWLEDGMENTS}

S.B.C. and J.M.H. thanks the Instituto do Milênio de Informação Quântica, CAPES, CNPq, FAPEAL, PRONEXNEON, ANP-CTPETRO for support. A.M.K. is grateful to FAPESP, FAPEAL, and RFBR (Grant No. 01-01 00696). R.A.K. gratefully acknowledges the support of FAPESP.
[1] A.I. Maimistov and E.A. Manykin Zh. Eksp. Teor. Fiz. 85, 1177 (1983) [Sov. Phys. JETP 58, 685 (1983)].

[2] M. Nakazawa, E. Yamada, and H. Kubota, Phys. Rev. Lett. 66, 2625 (1991); M. Nakazawa, E. Yamada, and H. Kubota, Phys. Rev. A 44, 5973 (1991).

[3] E.J.S. Fonseca, S.B. Cavalcanti, and J.M. Hickmann, Phys. Rev. E 64, 016610 (2001).

[4] S.B. Cavalcanti, A.M. Kamchatnov, R.A. Kraenkel, and B.A. Umarov, J. Mod. Opt. 49, 2183 (2002).

[5] M. Nakazawa, Y. Kimura, K. Kurokawa, and K. Suzuki, Phys.
Rev. A 45, R23 (1992).

[6] D. Anderson, Phys. Rev. A 27, 3135 (1983).

[7] S.C. Cerda, S.B. Cavalcanti, and J.M. Hickmann, Eur. Phys. J. D 1, 313 (1998).

[8] V.V. Steblina, Y.S. Kivshar, M. Lisak, and B.A. Malomed, Opt. Commun. 118, 345 (1995).

[9] V.M. Agranovich, S.A. Darmanyan, A.M. Kamchatnov, T.A. Leskova, and A.D. Boardman, Phys. Rev. E 55, 1894 (1997).

[10] J.A. Armstrong, Phys. Rev. A 11, 963 (1975). 